\title{
IL WRONSKIANO E LA DIPENDENZA LINEARE DI $n$ FUNZIONI DI UNA VARIABILE REALE.
}

\author{
Nota di Piero Martinotti (Pavia).
}

Adunanza del 9 febbrajo 1913.

Dopo che Peano ${ }^{2}$ ), con esempî, ha mostrato che non sempre l'annullarsi del Wronskiano di $n$ funzioni $f_{1}, f_{2}, \ldots, f_{n}$, per tutti i valori della variabile reale $x$ appartenenti ad un dato intervallo $(a b)$, è condizione sufficiente affinchè fra queste funzioni esista una stessa relazione lineare omogenea in $(a b)$, poche ricerche sono state fatte sopra questo argomento, onde brevemente possono essere tutte riassunte.

Peano ${ }^{2}$ ) e Vivanti ${ }^{3}$ ), con metodi differenti, hanno dimostrato che si ottengono condizioni sufficienti aggiungendo alla predetta l'ipotesi che i complementi algebrici degli elementi dell'ultima orizzontale del Wronskiano non si annullino tutti in uno stesso punto di $(a b)$.

Bôcher ${ }^{4}$ ) e Kowalewski ${ }^{5}$ ) hanno aggiunto una condizione diversa, ma che puó ritenersi più restrittiva di questa, e cioè che vi sia un complemento algebrico degli elementi dell'ultima orizzontale che non si annulli in nessun punto di $(a b)$. Il primo dei suddetti Autori ha poi ricavato varie generalizzazioni di questa condizione, delle quali la principale è data dal non annullarsi in nessun punto di $(a b)$ di un complemento algebrico degli elementi dell'ultima orizzontale assieme ad un certo numero di sue derivate successive.

In una Nota recente ${ }^{6}$ ) ho dimostrato che basta fare l'ipotesi che in $(a b)$ non vi siano punti zero comuni a tutti i complementi algebrici degli elementi di una orizzon-

I) G. Peano, Sur le déterminant Wronskien [Mathesis, rère série, t. IX (I889), pp. 75-76, I Io-I I2].

2) G. Peano, Sul determinante Wronskiano [Rendiconti della. R. Accademia dei Lincei (Roma), vol. VI, $\mathrm{I}^{\circ}$ semestre 1897 , pp. 4I3-4I 5 ].

3) G. Vivanti, Sul determinante Wronskiano [Rendiconti della R. Accademia dei Lincei (Roma), vol. VII, I ${ }^{\circ}$ semestre I898, pp. 194-197].

4) M. Bòcher, Certain cases in which the vanishing of the Wronskian is a sufficient condition for linear dependence [Transactions of the American Mathematical Society, vol. II (1901), pp. I39-149].

5) G. Kowalewski, Einfübrung in die Determinantentheorie, einschliesslich der unendlichen und Fredholmschen Determinanten (Leipzig, Veit \& Co., 1909), p. 327.

6) P. Martinotri, Sul Wronskiano [Rendiconti del R. Istituto Lombardo di Scienze e Lettere (Milano), serie II, vol. XLVI (1913), pp. 133-136]. 
tale qualunque. OrLando ${ }^{7}$ ) ha limitato lo studio a quei sistemi di $n$ funzioni tali che nessuna loro combinazione razionale presenti in $(a b)$ tratti continui di punti zero, senza esservi costantemente nulla, e per essi sistemi, con una lieve semplificazione sul procedimento di Vivantr, ha constatato la validità del teorema del Wronskiano senza limitazioni relative alle obbiezioni di Peano.

Qui ora mi sono proposto di deterninare le condizioni che, insieme a quella dell'annullarsi del Wronskiano, sono necessarie e sufficienti per l'esistenza di una relazione lineare omogenea fra $n$ funzioni in un dato intervallo, intendendo sempre, anche quando non venga espresso, che detta relazione sia la stessa per tutti $\mathrm{i}$ punti di quell'intervallo.

Credo che il tener conto di queste condizioni non costituisca un eccesso di rigore, perchè, come rilevo al $\ 3$, gli esempî di Peano non sono da riguardarsi come vere eccezioni, ma fanno parte di casi che, se non sono comuni nella pratica, si possono molto facilmente ottenere. Infine $(\$ 6)$ sono condotto al risultato che nei casi ordinari di funzioni sviluppabili in serie di TAYLOR si puó rigorosamente fare a meno delle condizioni medesime.

Siccome in questo studio interessa tener conto del numero delle derivate successive che ammettono le funzioni considerate, mi è tornato utile servirmi di una generalizzazione apportata da MLodzIEjowski ${ }^{8}$ ) al teorema del Wronskiano. Essa consiste nel considerare in luogo di questo un determinante avente le prime linee, in numero qualunque, formate con le derivate successive delle funzioni $f_{1}, f_{2}, \ldots, f_{n}$, e le altre con costanti arbitrarie, salvo una condizione che verrà precisata in seguito $(\S 4)$. MLodziejowski non si preoccupa peró affatto della questione alla quale é rivolto il presente studio.

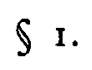

Nella predetta mia Nota ho posto in rilievo come, seguendo la dimostrazione di VIVANTI, la ricerca delle condizioni sotto le quali l'annullarsi del Wronskiano induce una dipendenza lineare fra le relative funzioni, è strettamente legata con la ricerca di certe condizioni affinchè $n$ funzioni $\varphi_{1}, \varphi_{2}, \ldots, \varphi_{n}$ in un certo intervallo $(a b)$, nel quale ammettono derivata, differiscano soltanto per un fattore costante, cioè soddisfino alla relazione:

$$
\frac{P_{1}}{c_{1}}=\frac{\varphi_{2}}{c_{2}}=\frac{\varphi_{3}}{c_{3}}=\cdots=\frac{\varphi_{n}}{c_{n}}=\psi(x),
$$

ove $c_{1}, c_{2}, \ldots, c_{n}$ sono costanti diverse da zero.

7) L. Orlando, Sul determinante di Wronski [Rendiconti della R. Accademia dei Lincei (Roma), vol. XVII, ${ }^{\circ}{ }^{\circ}$ semestre 1908 , pp. 7 17-720]. Nella prima parte di questa Nota e fatta una dettagliata esposizione della questione ivi e qui trattata.

8) B. K. MLodziejowski, Sur une generalisation du déterminant de Wronski [Recueil Mathématique de Moscou, t. XXV (1904-1905), pp. 474-477].

Rend. Circ. Matem. Palermo, t. XXXV ( ${ }^{\circ}$ sem. 1913). - Stampato il 26 marzo 1913. 
Nel caso di due funzioni questa relazione stabilisce fra di esse una dipendenza lineare omogenea, e quindi la ricerca predetta coincide con la questione del Wronskiano.

Vivanti ha dimostrato che condizioni sufficienti per la (I) sono:

$\mathrm{I}^{\mathrm{a}}$ che in tutto l'intervallo $(a b)$ abbia luogo la proporzione:

$$
\frac{\varphi_{1}^{\prime}}{\varphi_{1}}=\frac{\varphi_{2}^{\prime}}{\varphi_{2}}=\cdots=\frac{\varphi_{n}^{\prime}}{\varphi_{n}} ;
$$

$2^{2}$ che in nessun punto di $(a b)$ si annullino insieme le funzioni $\varphi$.

La prima di queste condizioni è anche necessaria, come è facile vedere, e perciò non vi è ragione di modificarla, tanto più che sotto tale forma serve per il nostro scopo. La seconda invece non è affatto necessaria, anzi restringe alquanto il campo di applicabilità di quelle condizioni, in quanto vengono esclusi tutti quei casi nei quali la $\psi(x)$ si annulla in qualche punto dell'intervallo $(a b)$.

Dico che a questa condizione si puó sostituire la seguente:

$3^{2}$ nei punti di $(a b)$ che annullano tutte le funzioni $\varphi$, i mutui rapporti di queste siano funzioni continue della $x$,

la quale, evidentemente, è anche necessaria per l'esistenza della relazione (I).

Basta, per la dimostrazione, considerare il caś di due sole funzioni, chè il passaggio al caso generale puó farsi senza varianti.

Le funzioni $\varphi_{1}(x)$ e $\varphi_{2}(x)$ che a tale scopo. consideriamo dovranno ammettere in ( $a b)$ almeno le derivate prime, affinchè possa aver luogo la prima condizione; ammetteremo di più che queste derivate siano continue, per modo che $\varphi_{1}$ e $\varphi_{2}$ non potranno avere infiniti punti zero in ogni porzione comunque piccola dell'intervallo considerato, senza esservi costantemente nulle ${ }^{9}$ ).

Ora, se queste funzioni soddisfano alla $I^{\text {a }}$ condizione, si sa che in ogni porzione di $(a b)$ non contenente valori di $x$ che le annullano entrambe, il rapporto $\frac{\phi_{1}(x)}{\varphi_{2}(x)}$ è costante; se questo rapporto è anche funzione continua di $x$ in $(a b)$, essendo costante in tutti $\mathrm{i}$ punti di questo intervallo, salvo i punti zero comuni alle due funzioni considerate, il cui insieme è limitato dall'ipotesi premessa, dovrà essere costante nell'intero intervallo considerato ${ }^{\text {ro }}$ ).

E poi chiaro che basterà assicurarsi della continuita di detto rapporto per $\mathrm{i}$ soli valori di $x$ che annullano tanto $\varphi_{1}$ che $\varphi_{2}$.

Riassumendo, si puó enunciare il teorema:

Affinche $n$ funzioni differiscano fra loro solo per un fattore costante in un intervallo nel quale banno derivata continua, è necessario e sufficiente che nell'intervallo stesso siano proporzionali alle rispettive derivate, e che nei punti nei quali esse diventano tutte nulle, $i$ loro mutui rapporti si mantengano funzioni continue.

9) U. DinI, Fondamenti per la teorica di variabili reali (Pisa, 1878), pag. I67.

זo) U. Dini, loc. cit. 9), pag. 73. 


\section{$\oint 2$.}

Se $x_{0}$ è un punto di $(a b)$ tale che

$$
\varphi_{1}\left(x_{0}\right)=\varphi_{2}\left(x_{0}\right)=0,
$$

affinchè $\frac{\varphi_{1}(x)}{\varphi_{2}(x)}$ sia continua per $x=x_{0}$ è necessario e sufficiente che essa funzione tenda ad un limite determinato col tendere di $x$ ad $x_{0}$.

Ciò accade se le derivate di $\varphi_{1}(x)$ e $\varphi_{2}(x)$, di un ordine qualunque $r$, sempre mantenendosi continue per $x=x_{o}$, vi assumono un valore diverso da zero, poichè allora, applicando successivamente la regola di L'Hôpital ${ }^{11}$ ), si ha :

$$
\lim _{x=x_{0}} \frac{\varphi_{1}(x)}{\varphi_{2}(x)}=\lim _{x=x_{0}} \frac{\varphi_{1}^{(r)}(x)}{\varphi_{2}^{(r)}(x)}=\frac{\varphi_{1}^{(r)}\left(x_{0}\right)}{\varphi_{2}^{(r)}\left(x_{0}\right)} .
$$

Questa condizione non è però necessaria: lo constatiamo con l'esempio:

$$
\varphi_{1}(x)=c x|x|, \quad \varphi_{2}(x)=x|x|
$$

ove $c$ è una costante diversa da zero, $\mathrm{e}|x|$ denota il valore assoluto di $x$. Nell'intervallo $(-\infty,+\infty)$ queste funzioni non ammettono che le derivate prime:

$$
\varphi_{1}^{\prime}(x)=2 c|x|, \quad \varphi_{2}^{\prime}(x)=2|x|
$$

le quali sono continue, ma non derivabili per $x=0$, si annullano, come le relative funzioni, per $x=0$, eppure il rapporto $\frac{\varphi_{1}(x)}{\varphi_{2}(x)}$ è funzione continua in tutto quell'intervallo.

Potrebbe sembrare, nell'intento di trovare una condizione praticamente più semplice della $3^{\text {a }}$, che bastasse l'ipotesi, evidentemente necessaria per la (I), che il rapporto

II) Se si tien conto della $I^{\text {a }}$ ipotesi si perviene a questo risultato anche senza la regola di L'HóPITAL, poichè da quella, ossia dall'identità :

si puó dedurre la seguente:

$$
\frac{\varphi_{1}(x)}{\varphi_{2}(x)}=\frac{\varphi_{1}^{\prime}(x)}{\varphi_{2}^{\prime}(x)}
$$

qualunque sia l'ordine di derivazione $r$.

$$
\frac{\varphi_{1}(x)}{\varphi_{2}(x)}=\frac{\varphi_{1}^{(r)}(x)}{\varphi_{2}^{(r)}(x)}
$$
niamo sia :

Essa sussiste infatti, per ipotesi, nel caso di $r=\mathrm{I}$; procedendo per induzione completa, suppo(b)

$$
\varphi_{1} \xi_{2}^{(r-1)}=\psi_{1}^{(r-1)} \varphi_{2}
$$

derivando:

ma dalle (a) e (b) si ricava :

$$
\varphi_{1}^{\prime} \varphi_{2}^{(r-1)}+\varphi_{1} \varphi_{2}^{(r)}=\varphi_{1}^{(r)} \varphi_{2}+\varphi_{1}^{(r-1)} \varphi_{2}^{\prime}
$$

onde la precedente diventa:

$$
\varphi_{1}^{\prime} \varphi_{2}^{(r-1)}=\varphi_{1}^{(r-1)} \varphi_{2}^{\prime} \text {, }
$$

che è l'uguaglianza da dimostrarsi.

$$
\varphi_{1} \varphi_{2}^{(r)}=\varphi_{1}^{(r)} \varphi_{2},
$$


$\frac{\varphi_{1}^{(r)}\left(x_{0}\right)}{\varphi_{2}^{(r)}\left(x_{0}\right)}$, per un certo ordine di derivazione $r$, sia determinato, pur potendo esser nulli tanto il numeratore che il denominatore, per la presenza di un fattore, funzione di $x$, annullantesi per $x=x_{\mathrm{o}}$. Che ciò non sia, come è teoricamente prevedibile, risulta dal primo degli esempî di Peano:

$$
\varphi_{1}(x)=x^{2}[\mathrm{1}+f(x)], \quad \varphi_{2}(x)=x^{2}[\mathrm{r}-f(x)],
$$

ove $f(x)$ designa una funzione nulla per $x=0$, uguale a I per $x>0$ ed a - I per $x<0$. Queste funzioni ammettono, per qualsiasi valore di $x$, solo le derivate prime:

$$
\varphi_{1}^{\prime}(x)=2 x[\mathrm{I}+f(x)], \quad \varphi_{2}^{\prime}(x)=2 x[\mathrm{I}-f(x)]
$$

pure continue, ma non derivabili per $x=0$. Esse soddisfano alla $\mathrm{I}^{\mathrm{a}}$ condizione, inoltre si ha:

da cui:

$$
\frac{\phi_{1}^{\prime}(x)}{\varphi_{2}^{\prime}(x)}=\frac{1+f(x)}{I-f(x)}
$$

$$
\frac{\varphi_{1}^{\prime}(0)}{\varphi_{2}^{\prime}(0)}=\frac{I+f(0)}{I+f(0)}=\mathrm{I}
$$

eppure il loro rapporto non è costante.

Ma la condizione, solo sufficiente, indicata in principio di questo $\$, può rendere utili servigi, poichè è facile verificare che essa è soddisfatta quando $\phi_{1}$ e $\varphi_{2}$ siano sviluppabili in serie di TAYLOR nell'intervallo considerato; infatti, essendo da escludersi che dette funzioni siano identicamente nulle nell'intervallo stesso, dovranno le loro derivate di un certo ordine risultare diverse da zero per $x=x_{0}$. Ciò è valido anche per un numero qualunque di funzioni, quindi:

Condizione necessaria e sufficiente affinchè piu funzioni sviluppabili in serie di TAYLOR differiscano solo per un fattore costante è che siano proporzionali alle rispettive derivate.

$\$ 3$.

Tornando al caso generale trattato al $\ \mathrm{I}$, e riferendomi alle obbiezioni di Peano, voglio indicare un modo semplice per costruire degli esempî che provano l'insufficienza della $\mathrm{I}^{\mathrm{a}}$ condizione ivi enunciata.

Basta prendere per $\varphi_{1}(x)$ una funzione avente derivata continua in un intervallo $(a b)$ nel quale presenta un solo punto zero $x_{0}$, ma del resto qualunque; per $\varphi_{2}(x)$ la funzione che per $x<x_{0}$ è uguale al prodotto di $\varphi_{1}(x)$ per una costante qualunque diversa da zero, per $x=x_{0}$ è uguale a o, e per $x>x_{0}$ al prodotto di $\varphi_{1}(x)$ per una costante, ancora non nulla, ma diversa dalla prima.

Come caso particolare si ha il seguente esempio di Peano:

$$
\begin{gathered}
\varphi_{1}(x)=x^{2}, \\
\rho_{2}(x)=x|x|=\left\{\begin{array}{ccc}
-x^{2} & \text { per } x<0, \\
0 & \Rightarrow & x=0, \\
x^{2} & \Rightarrow & x>0 .
\end{array}\right.
\end{gathered}
$$


Relativamente al primo esempio, gid considerato nel $\$$ precedente, è poi evidente che due funzioni qualunque derivabili nell'intervallo $(a b)$ e tali che l'una sia nulla in tutti i punti di una sua parte $(a c)$ e l'altra lo sia invece nella rimanente parte $(c b)$, soddisfano sempre alla prima condizione in tutto $(a b)$ senza che in questo intervallo il loro rapporto possa essere costante.

\section{$\int 4$.}

Veniamo ora allo studio della dipendenza lineare di $n$ funzioni $f_{1}, f_{2}, \ldots, f_{n}$ in un certo intervallo $(a b)$, escludendo quelle funzioni che vi fossero identicamente nulle.

Supponiamo che nell'intervallo stesso le $f$ ammettano derivate successive sino ad ordini diversi, e sia $m$ il più piccolo di tutti questi ordini; formiamo la matrice:

$$
\left|\begin{array}{cccc}
f_{1} & f_{2} & \cdots & f_{n} \\
f_{1}^{\prime} & f_{2}^{\prime} & \cdots & f_{n}^{\prime} \\
f_{1}^{\prime \prime} & f_{2}^{\prime \prime} & \cdots & f_{n}^{\prime \prime} \\
\cdots & \cdots & \cdots \cdot & f_{n}^{(m)}
\end{array}\right|
$$

Consideriamo in questa un numero $b$ di orizzontali contate dalla prima, tale che nella matrice formata con le prime $b-$ I di esse non vi sia alcun minore di ordine $b$ - I che in $(a b)$ sia identicamente nullo. Si potrà, in generale, soddisfare a questa condizione con diversi valori di $b$ : supponiamo di fissarne uno che sia minore di $n$.

Alle suddette $b$ linee aggiungiamone altre $n-b$ aventi per elementi delle costanti, ottenendo cosi il determinante:

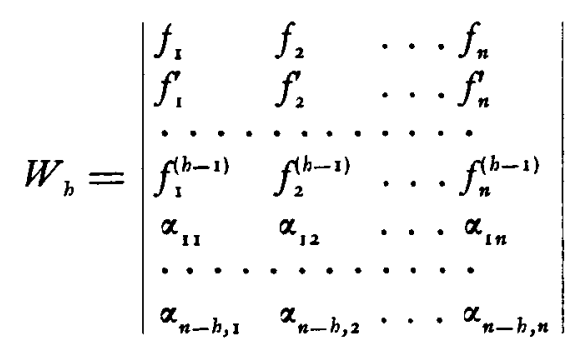

e le costanti $\alpha$ debbono essere tali che nella matrice risultante da questo determinante sopprimendo la $b$-esima orizzontale vi sia un minore di ordine $n$ - I non identicamente nullo nell'intervallo $(a b)$.

Uno dei modi più semplici per ottenere questo, consisterà nel fissare le costanti $\alpha$ in guisa che la loro matrice contenga un solo minore d'ordine $n-b$ avente un valore $D \neq 0$, e gli altri tutti nulli; allora se si prende in $W_{h}$ un minore d'ordine $n$ - I sopprimendo la $b$-esima orizzontale ed una verticale non comprendente elementi di $D$, sviluppando con la regola di LAPLACE questo minore, ove si separino le prime $b-$ I orizzontali dalle rimanenti, si vede che esso è uguale al prodotto di $D$ per il suo complemento algebrico, e quindi, per ipotesi, non identicamente nullo. 
Il determinante $W_{h}$ gode di una proprieta comune con il Wronskiano:

Per tutti $i$ valori $d i x$ che annullano $W_{b}$, $i$ complementi algebrici degli elementi di una sua linea qualunque sono proporzionali alle rispettive derivate.

Infatti ${ }^{\mathbf{r}^{2}}$ ), indicando con $D_{1}, D_{2}, \ldots, D_{n}$ i complementi algebrici di una orizzontale qualsiasi, dall'ipotesi $W_{b}=0$ si deducono le relazioni :

$$
\begin{aligned}
& D_{1} f_{1}+D_{2} f_{2}+\cdots+D_{n} f_{n}=0, \\
& D_{1} f_{1}^{\prime}+D_{2} f_{2}^{\prime}+\cdots+D_{n} f_{n}^{\prime}=0, \\
& \cdots \cdots+D_{n} f_{2}^{(h-1)}+\cdots+D_{n} f_{n}^{h-1)}=0, \\
& D_{1} f_{1}^{(h-1)}+\cdots+D_{n} \alpha_{1 n}=0, \\
& D_{1} \alpha_{11}+D_{2} \alpha_{12}+\cdots \cdots+\cdots \\
& D_{1} \cdots \cdots D_{n} \alpha_{n-h, n}=0 .
\end{aligned}
$$

Derivandole tutte, salvo la $b$-esima, e tenendo conto delle relazioni stesse, si ricavano le:

$$
\begin{aligned}
& D_{1}^{\prime} f_{1}+D_{2}^{\prime} f_{2}+\cdots+D_{n}^{\prime} f_{n}=0 \text {, } \\
& D_{1}^{\prime} f_{1}^{\prime}+D_{2}^{\prime} f_{2}^{\prime}+\cdots+D_{n}^{\prime} f_{n}^{\prime}=\text {, } \\
& D_{1}^{\prime} f_{1}^{(h-2)}+D_{2}^{\prime} f_{2}^{(h-2)}+\cdots+D_{n}^{\prime} f_{n}^{(h-2)}=0 \text {, } \\
& D_{1}^{\prime} \alpha_{11}+D_{2}^{\prime} \alpha_{12}+\cdots+D_{n}^{\prime} \alpha_{1 n}=0 \text {, } \\
& D_{1}^{\prime} \alpha_{n-h, 1}+D_{2}^{\prime} \alpha_{n-h, 2}+\cdots+D_{n}^{\prime} \alpha_{n-h, n}=0 .
\end{aligned}
$$

Da questo sistema di $n$ - I equazioni lineari omogenee con le $n$ incognite $D_{1}^{\prime}$, $D_{2}^{\prime}, \ldots, D_{n}^{\prime}$ e dal precedente, meno la $b$-esima, con le incognite $D_{1}, D_{2}, \ldots, D_{n}$, poichè la matrice comune dei coefficienti ha, per le ipotesi fatte nella costruzione di $W_{b}$, caratteristica $n-\mathrm{I}$, si deduce l'asserto:

$$
D_{1}^{\prime}: D_{2}^{\prime}: \cdots: D_{n}^{\prime}=D_{1}: D_{2}: \cdots: D_{n} \text {. }
$$

$\$ 5$.

Ciò posto, si ha il teorema:

Affinche fra $n$ funzioni esista una relazione lineare omogenea in un intervallo ( $a b$ ) nel quale ammettono derivata continua, è necessario e sufficiente che si possa costruire un determinante $W_{b}(b \supseteq 2)$ nullo in tutti $i$ punti di detto intervallo, e che in quelli fra essi punti che annullano tutti $i$ complementi algebrici degli elementi di una orizzontale qualunque di $W_{b}$, i rapporti di questi complementi algebrici siano funzioni continue.

12) Il procedimento che segue potrebbe applicarsi per dimostrare la proprietà analoga del Wronskiano, e ciò con vantaggio di semplicità sulla dimostrazione che ne ho già dato [loc. cit. ${ }^{6}$ )]. 
Infatti, se esiste la relazione:

$$
c_{1} f_{1}+c_{2} f_{2}+\cdots+c_{n} f_{n}=0
$$

e le $f$, oltre alla prima, ammettono derivate successive sino ad ordini qualunque, si potranno avere per derivazione altre $b-1$ relazioni:

$$
c_{\mathrm{v}} f_{\mathrm{r}}^{\prime}+c_{2} f_{2}^{\prime}+\cdots+c_{n} f_{n}^{\prime}=\mathrm{o}
$$

tali che nessuno dei minori d'ordine $b$ - I contenuti nella matrice:

$$
\left|\begin{array}{llll}
f_{1} & f_{2} & \cdots & f_{n} \\
f_{1}^{\prime} & f_{2}^{\prime} & \cdots & f_{n}^{\prime} \\
\cdots & \cdots & \cdots & \cdots \\
f_{1}^{(h-2)} & f_{2}^{(h-2)} & \cdots & f_{n}^{(h-2)}
\end{array}\right|
$$

sia nullo nell'intero intervallo $(a b)$. Se poi si considera l'equazione lineare omogenea :

$$
c_{1} x_{1}+c_{2} x_{2}+\cdots+c_{n} x_{n}=0
$$

sarà sempre possibile determinare $n-b$ sue soluzioni:

$$
\begin{aligned}
& \alpha_{11}, \quad \alpha_{12}, \ldots, \alpha_{1 n} \\
& \ldots \ldots . \ldots, \alpha_{n-h, n}
\end{aligned}
$$

che soddisfino alle condizioni volute per formare, di seguito alle derivate delle $f$, il determinante $W_{b}$, poichè i valori di $n-1$ fra le incognite $x_{1}, x_{2}, \ldots, x_{n}$ si possono fissare ad arbitrio.

Allora $c_{1}, c_{2}, \ldots, c_{n}$ si possono considerare come soluzioni non nulle del sistema di $n$ equazioni lineari omogenee avente per determinante dei coefficienti $W_{b}$ :

$$
\begin{aligned}
& c_{1} f_{1}+c_{2} f_{2}+\cdots+c_{n} f_{n}=0, \\
& c_{1} f_{1}^{\prime}+c_{2} f_{2}^{\prime}+\cdots+c_{n} f_{n}^{\prime}=0 \text {, }
\end{aligned}
$$

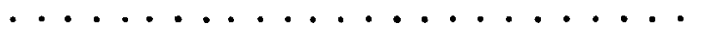

$$
\begin{aligned}
& c_{1} f_{\mathrm{I}}^{(h-1)}+c_{2} f_{2}^{(h-1)}+\cdots+c_{n} f_{n}^{(h-1)}=0 \text {, } \\
& c_{1} \alpha_{11}+c_{2} \alpha_{12}+\cdots+c_{n} \alpha_{1 n}=0 \text {, } \\
& \ldots \ldots \ldots \ldots \ldots \ldots \\
& c_{1} \alpha_{n-h, 1}+c_{2} \alpha_{n-h, 2}+\cdots+c_{n} \alpha_{n-h, n}=0
\end{aligned}
$$

e da questo si ricava la prima condizione $W_{b}=0$.

Siccome poi $W_{h}$ ha caratteristica $n-1$, indicando ancora con $D_{1}, D_{2}, \ldots, D_{n}$ i complementi algebrici degli elementi di una sua orizzontale qualunque, si ha:

$$
c_{1}: c_{2}: \cdots: c_{n}=D_{1}: D_{2}: \cdots: D_{n}
$$

per cui $(\$ \mathrm{I})$ se esistono punti di $(a b)$ nei quali si annullano contemporaneamente $D_{1}, D_{2}, \ldots, D_{n}$, in essi dovranno essere funzioni continue della $x$ i mutui rapporti delle $D$.

Con ciò la necessità delle condizioni del teorema resta dimostrata.

Viceversa, dall'ipotesi $W_{h}=0$ si deduce $(\$ 4)$ :

$$
D_{1}^{\prime}: D_{2}^{\prime}: \cdots: D_{n}^{\prime}=D_{1}: D_{2}: \cdots: D_{n}
$$


e per la continuità dei rapporti di $D_{1}, D_{2}, \ldots, D_{n}$ nei punti ove queste funzioni si annullano ad un tempo, si ha $\left(\int_{1}\right)$ :

$$
\frac{D_{1}}{c_{1}}=\frac{D_{1}}{c_{2}}=\cdots=\frac{D_{n}}{c_{n}}=\psi(x) \text {. }
$$

D'altra parte, ancora per l'annullarsi di $W_{h}$, esiste l'identità :

$$
D_{1} f_{1}+D_{1} f_{2}+\cdots+D_{n} f_{n}=\mathrm{o},
$$

da cui per la precedente:

$$
c_{1} f_{\mathrm{s}}+c_{2} f_{2}+\cdots+c_{n} f_{n}=\mathrm{o}
$$

$\$ 6$.

Il teorema del Wronskiano corrisponde al caso particolare di $b \supseteq n$, e puó enunciarsi :

Condizione necessaria e sufficiente affinchè $n$ funzioni, in un intervallo (ab) nel quale ammettono almeno le prime $n$ derivate successive, non siano linearmente indipendenti, $\dot{e}$ che il Wronskiano di dette funzioni si annulli identicamente nell'intervallo stesso, o che nei punti di questo che annullano contemporaneamente tutti i complementi algebrici degli elementi di una orizzontale qualunque di detto determinante, $i$ rapporti di questi complementi algebrici siano funzioni continue.

Se le $n$ funzioni considerate sono in ( $a b)$ sviluppabili in serie di TayLor, tutti $\mathrm{i}$ minori di ordine $n$ - I del relativo Wronskiano saranno pure sviluppabili in serie nello stesso intervallo. Allora, siccome la continuitd dei loro rapporti è assicurata, come si vide al $\int 2$, si deduce il seguente risultato che interessa i casi più comuni:

Per le funzioni sviluppabili in serie di TAYLOR l'annullarsi del Wronskiano è condizione necessaria e sufficiente affinchè siano legate da una relazione lineare omogenea a coefficienti costanti non nulli.

\section{$\S 7$.}

Termino con una osservazione che riguarda la dimostrazione data da Demoulin ${ }^{\mathbf{3}}$ ) del teorema del Wronskiano, riportata in molti trattati, e dalla quale risulta il semplice annullarsi di detto determinante condizione necessaria e sufficiente per la dipendenza lineare di funzioni $n-\mathrm{I}$ volte derivabili. Tale dimostrazione, procedente per induzione completa, non lascerebbe adito ad alcuna obbiezione, ma è fondata su una nota proprietà dei Wronskiani che non è sempre applicabile, almeno senza l'aggiunta di speciale condizione. Voglio dire la proprietà comune con le funzioni omogenee:

13) A. Demoulin, Démonstration de la proprieté fondamentale des Wronskiens [Mathesis, $2^{e}$ série, t. VII (1897), pp. 62-63]. 
Se si moltiplicano le funzioni $f_{1}, f_{2}, \ldots, f_{n}$ per una funzione $\varphi$, il loro Wronskiano resta moltiplicato per $\varphi^{n}$.

Puó darsi che per qualche valore particolare della variabile qualcuno dei prodotti $\varphi f_{1}, \varphi f_{2}, \ldots, \varphi f_{n}$ non ammetta tutte le $n-\mathrm{x}$ derivate necessarie per formarne il relativo Wronskiano, ed allora, in intervalli comprendenti i punti stessi, quella proprietà perde significato.

E ciò che accade per l'esempio di PeAno:

$$
f_{1}(x)=x^{2} \quad f_{2}(x)=x|x|
$$

se si moltiplicano queste funzioni per $\frac{\mathrm{r}}{x^{2}}$. Dalla seconda si ha:

$$
\frac{I}{x^{2}} f_{2}(x)=\frac{|x|}{x},
$$

che non è derivabile in intervalli comprendenti l'origine.

Pavia, Febbraio 1913.

Piero Martinotiti. 